\title{
Improving Multiple Health Risk Behaviors in Primary Care: Lessons from the Prescription for Health COmmon Measures, Better Outcomes (COMBO) Study
}

\author{
Douglas H. Fernald, MA, L. Miriam Dickinson, PhD, Desireé B. Froshaug, MS, \\ Bijal A. Balasubramanian, MBBS, PhD, Jodi Summers Holtrop, PhD, \\ Alex H. Krist, MD, MPH, Russell E. Glasgow, PhD, and Larry A. Green, MD
}

Background: Four health behaviors—smoking, risky drinking, physical inactivity, and unhealthy diets-contribute substantially to health care burden and are common among primary care patients. However, there is insufficient evidence to recommend broadly brief interventions to address all 4 of these in frontline primary care. This study took advantage of a multinetwork initiative to reflect on health behavior outcomes and the challenges of using a common set of measures to assess health behavior-change strategies for multiple health behaviors in routine primary care practice.

Methods: Standardized, brief practical health behavior and quality of life measures used across 7 practice-based research networks (PBRNs) with independent primary care interventions in 54 primary care practices between August 2005 and December 2007 were analyzed. Mixed-effects longitudinal models assessed whether intervention patients improved diet, physical activity, smoking, alcohol consumption, and unhealthy days over time. Separate analyses were conducted for each intervention.

Results: Of 4463 adults, 2199 had follow-up data, and all available data were used in longitudinal analyses. Adjusting for age, race/ethnicity, education, and baseline body mass index where available, diet scores improved significantly in 5 of 7 networks $(P<.02)$. Physical activity improved significantly in 2 networks but declined in one network $(P<.024)$. The likelihood of being a current smoker was reduced in 2 of 5 networks $(P<.0001)$, and average alcoholic drinks per day was reduced in 2 network $s(P<.02)$. Participants reported fewer unhealthy days at follow-up in 3 of 7 networks $(P<.01)$. Details of implementation and the limitations in instrumentation help contextualize these modest outcomes.

Conclusions: Although some patients in these 7 PBRNs improved in several health behaviors and quality of life, the strength of evidence for field-ready methods to address multiple health risk behaviors remains elusive. The use of common measures to assess changes in 4 unhealthy behaviors was achieved practically in PBRNs testing diverse strategies to improve behaviors; however, variations in implementation, instrumentation performance, and some features of study design overwhelmed potential cross-PBRN comparisons. For common measures to be useful for comparisons across practices or PBRNs, greater standardization of study designs and careful attention to practicable implementation strategies are necessary. (J Am Board Fam Med 2012;25:701-711.)

Keywords: Alcohol Drinking, Crisis Intervention, Counseling, Diet, Health Behavior, Physical Activity, PracticeBased Research, Practice-Based Research Networks, Primary Health Care, Risk Behavior, Smoking

Despite efforts to curb unhealthy diets, to increase physical activity, to reduce smoking, and to limit risky drinking, a substantial proportion of US citi-

This article was externally peer reviewed.

Submitted 15 February 2011; revised 16 December 2011; accepted 3 January 2012. zens require improvement in these areas. Primary care offices in the United States have been characterized as underutilized sites for routine screening of

From the Department of Family Medicine, University of Colorado, Aurora (DHF, LMD, LAG); the Colorado Foundation for Medical Care, Englewood (DBF); the University 
patients for the multiple health risk behaviors of lowquality diets, physical inactivity, cigarette smoking, and risky drinking. ${ }^{1-5}$ Although it has been suggested that primary care practices could contribute significantly if they routinely addressed these health behaviors, which contribute to chronic disease and cancer, ${ }^{5,6}$ it is unclear if wider deployment of successful strategies will lead to improved health behaviors over time. A growing literature about efforts to address these multiple health behaviors simultaneously or sequentially has yielded modestly successful results, ${ }^{8-14}$ and there is a need for more information about real-world applications. ${ }^{16,17}$

Previous work confirmed that unhealthy behaviors persist in primary care populations, consistent with national surveillance data. In a sample of 67 primary care practices, $96.8 \%$ of adult patients had at least one unhealthy behavior and $69.2 \%$ had 2 or more unhealthy behaviors of unhealthy diet, underactivity, or smoking. ${ }^{17}$ However, it is not known if more widely implemented primary care interventions would result in meaningful changes to practical measures of health behaviors or quality of life. Prescription for Health was an initiative designed to address 4 health behaviors through innovative and practical strategies that was conceived and implemented independently by 10 practice-based research networks (PBRNs). Strategies included interventions such as practice redesign, new staff roles, connections to community resources, and in-office counseling.

In Prescription for Health, multiple PBRNs shared longitudinal health behavior and quality of life data from their projects that could be examined to determine if any changes were detectable in patients who received health behavior counseling interventions. This study took advantage of the multinetwork initiative to (1) report on important health behavior outcomes assessed by a common set of measures and (2) reflect on the challenges of

of Texas Southwestern Medical Center, Dallas (BAB); the Department of Family Medicine, Michigan State University, East Lansing (JSH); the Virginia Commonwealth University, Department of Family and Community Medicine, Richmond (AHK); and the Division of Cancer Control and Population Sciences, National Cancer Institute, Rockville, MD (REG).

Funding: Funding provided by The Robert Wood Johnson Foundation.

Conflict of interest: none declared.

Corresponding author: Douglas H. Fernald, MA, Mail Stop F496, 12631 E 17th Ave, Room 3423, Aurora, CO 80045 (E-mail: doug.fernald@ucdenver.edu). studying multiple health behavior change strategies in routine primary care practices across multiple research networks.

\section{Methods}

\section{Setting and Design}

Prescription for Health focused on 4 leading health risk behaviors associated with premature deathunhealthy diet, physical inactivity, tobacco use, and risky alcohol use-in 2 rounds of funding. ${ }^{18}$ Round 2 of the program funded 10 PBRNs to test further interventions to improve health behavior change in these 4 target behaviors among primary care patients. ${ }^{17}$ Nine PBRNs collected adult data, and 7 included follow-up patient contacts that could be used for evaluation of change in patient behaviors. (One PBRN did not submit timely follow-up data; another did not have longitudinal follow-up data because of its study design. Another PBRN collected only adolescent data.) Data were collected between August 2005 and December 2007. The Prescription for Health National Program Office formalized the study as Common Measures, Better Outcomes (COMBO) and assumed responsibility for combining and analyzing the combined data. The COMBO study was approved by the Colorado Multiple Institutional Review Board. Individual PBRN projects were approved by their respective human subjects review boards.

Each PBRN designed and implemented its own project, with differing interventions, target populations, data collection methods, and durations. Conceptual standardization was enhanced by requiring that all interventions address the Chronic Care Model $^{19}$ and consider how to address a planning, evaluation, and reporting framework known as RE-AIM (reach, effectiveness, adoption, implementation, and maintenance). ${ }^{20}$ Patient follow-up intervals from baseline were established by each PBRN and ranged from 4 weeks to 11 months. Despite their design differences, the projects shared several important elements: they addressed all 4 health behaviors, they assessed and monitored these health behaviors using common measures, and they linked patients to counseling resources (either within or outside of the practice) (Table 1). However, because of substantially different study designs and interventions, this analysis was not able to compare directly the effectiveness of different interventions. 
Table 1. Summary Description of the Multiple Health Behavior Interventions, Health Risk Data Collection Methods, and Study Designs

\begin{tabular}{|c|c|}
\hline PBRN ID & Intervention Description \\
\hline 1 & $\begin{array}{l}\text { Practices collaborated with a local health department to use an extension agent model to promote screening, } \\
\text { counseling, and use of community resources. } \\
\text { Intervention: used IT, new/modified staff roles, and counseling outside the practice } \\
\text { HRA: self-administered by computer } \\
\text { Design: longitudinal; no comparison group }\end{array}$ \\
\hline 2 & $\begin{array}{l}\text { A community health educator referral liaison was available to practices to provide patients with health } \\
\text { behavior counseling, follow-up, and assistance in connecting to community resources. } \\
\text { Intervention: used new/modified staff roles and counseling outside the practice } \\
\text { HRA: administered by liaison } \\
\text { Design: group (practice) randomized; control received physician referral only }\end{array}$ \\
\hline 3 & $\begin{array}{l}\text { Family screening assessments were conducted during modified well visits for children aged } 2 \text { years, and } \\
\text { families were directed to health behavior counseling. } \\
\text { Intervention: used new/modified staff roles and counseling outside the practice } \\
\text { HRA: administered by nurse staff as part of screening tool } \\
\text { Design: quasi-experimental; practices were assigned to groups }\end{array}$ \\
\hline 4 & $\begin{array}{l}\text { Patients were connected to web-based tools that promoted health behavior change accompanied by an IVR } \\
\text { telephone system to prompt website use. } \\
\text { Intervention: used IT, population screening/outreach, and counseling outside the practice } \\
\text { HRA: self-administered by computer } \\
\text { Design: patient randomized; control received usual care }\end{array}$ \\
\hline 5 & $\begin{array}{l}\text { Practice enhancement assistants provided performance feedback, training, practice change facilitation, and } \\
\text { local quality improvement collaboratives for behavior change. } \\
\text { Intervention: used new/modified staff roles, population screening/outreach, and counseling outside the } \\
\text { practice } \\
\text { HRA: self-administered by paper forms } \\
\text { Design: practices randomized to receive different sets of health behavior-specific modules in time-ordered } \\
\text { blocks }\end{array}$ \\
\hline 8 & $\begin{array}{l}\text { Existing medical assistants identified patients at risk for unhealthy behaviors then offered counseling and } \\
\text { referred to community resources. } \\
\text { Intervention: used new/modified staff roles, population screening/outreach, and counseling outside the } \\
\text { practice } \\
\text { HRA: self-administered by paper forms } \\
\text { Design: patient randomized; control received usual care. }\end{array}$ \\
\hline 9 & $\begin{array}{l}\text { Patients connected to an IVR telephone system to promote health behavior change and deliver counseling. } \\
\text { Intervention: used IT and counseling outside the practice } \\
\text { HRA: self-administered via the IVR system } \\
\text { Design: patient randomized; control received only printed educational materials }\end{array}$ \\
\hline
\end{tabular}

Adapted from Etz et al (2008) $)^{20}$ and Dodoo et al (2008). ${ }^{21}$

HRA, health risk assessment; IT, information technology; IVR, interactive voice recognition; PBRN, practice-based research network.

\section{Independent Variables}

Demographic characteristics were designed to be consistent with the Behavioral Risk Factor Surveillance System; included sex, age, income, and education; and were available in English or Spanish. ${ }^{23}$ Baseline weight and height measurements were used to calculate body mass index.

\section{Outcomes}

Based on previous work by Glasgow et $\mathrm{al}^{24}$ and feedback from the PBRNs, a set of 21 questions comprised the adult assessment tool for the health behaviors. ${ }^{18,20}$ The final set of questions were slightly modified from those recommended by Glasgow et $\mathrm{al}^{24}$ but were agreed on by all the investigators in Prescription for Health. (See Fernald et $\mathrm{al}^{17}$ for the full text of the questions used.) All questions were available in English or Spanish.

\section{Diet}

The diet instrument asked about 7 food habits: fast food, fruits/vegetables, sweet drinks, protein, chips/ crackers, desserts, and fats eaten during the last 7 days. ${ }^{25}$ Ordinal values were assigned to each response range. Summing the values provided a score of 0 to 14 , where 14 represented the least healthy diet habits. Imputation for up to 2 of the 7 missing dietary questions was implemented, substituting the mean of the completed responses for that indi- 
vidual. ${ }^{25}$ A reduction in the summed diet score was considered an improvement.

\section{Physical Activity}

The International Physical Activity Questionnaire (short version) was used to measure physical activity through metabolic equivalent-minutes, a continuous, universal, weighted sum of weekly exercise of different intensities and durations. ${ }^{26}$ Respondents were asked about time spent being physically active during the past 7 days and were classified as underactive if they had fewer than 5 days of at least 30 minutes of vigorous or moderate physical activity per week. Change in underactive status was evaluated.

\section{Smoking}

Questions about smoking asked if patients had ever smoked at least 100 cigarettes in their entire life and if they had smoked in the past 7 or 30 days. Those who smoked at least 100 cigarettes in their lifetime and smoked within the past 30 days at baseline were considered a current smoker. Both change in smoking status and number of cigarettes per day were assessed.

\section{Alcohol}

Questions from the Behavioral Risk factor Surveillance System 2003 were used to assess alcohol consumption in the past 30 days. If patients drank on at least 1 day in the past week or month, they were asked for the average number of drinks consumed on those days. An average number of drinks per day was calculated for those who had at least one drink in the past 30 days. Change in average drinks per day was assessed.

\section{Quality of Life}

Patients' perceived health-related quality of life (HRQOL) was captured through the Centers for Disease Control and Prevention's Healthy Days core measures (4-item HRQOL). ${ }^{27}$ These questions asked separately about the number of days during the past 30 that respondents felt physical and mental illness or injury. Summing these values and truncating at 30 days as described by the Centers for Disease Control and Prevention's guidelines provided the overall unhealthy days outcome. Change in number of unhealthy days was assessed.

\section{Statistical Analyses}

After an initial exploration of the data that examined characteristics of interventions associated with success, it was determined that the populations studied, research designs, and interventions were sufficiently diverse that meta-analyses were not appropriate. Thus, separate analyses were conducted for each PBRN. Descriptive statistics and frequency distributions were generated for patient characteristics; $t$ tests and $\chi^{2}$ tests were used to identify differences between patients who had follow-up data and who did not have any follow-up contact. Statistical analysis was performed using SAS software (version 9.2 for personal computer, SAS, Inc., Cary, NC).

Five sets of analyses for each PBRN were conducted to assess whether patients who received the intervention improved over time on diet, physical activity, smoking, alcoholic drinks per day, and unhealthy days. Three-level, hierarchical, mixed effects models using all available data and adjusted for clustering of observations within patients and patients within practices were used to assess baseline to follow-up change in patient-reported outcomes. ${ }^{28}$ For one PBRN (PBRN 3) there were too few practices to estimate practice random effects, so practice was included in the model as a fixed effect with only patient random effects. When comparison or control groups were available, separate analyses were conducted to examine change in outcomes over time along with a direct comparison of change by study group. Patient covariates that were significant at $P<.2$ in bivariate associations for any PBRN on any outcome or associated with dropout were included in all analyses (age, sex, education, and race/ethnicity). For each patient, baseline (time 0) was established as the day of the initial patient contact.

Although average time to follow-up is reported for all PBRNs, this study used a before/after approach to analyze data within each PBRN based on baseline and final observations. Among subjects with follow-up data, $89 \%$ had a total of 2 observations and $11 \%$ had 3 observations; for the latter only the first and last observations were used in analysis. Because of the high dropout rates in the individual PBRNs, sensitivity analyses were performed, assuming no improvement in patients who did not have follow-up data. These analyses also used a before/after approach on the basis of the first and last observations for each patient, with a last value carried forward imputation for patients without follow-up. 


\section{Results}

\section{Study Population}

A total of 4463 adult patients from 54 practices in 7 PBRNs were included in the study; 3768 patients were assigned to receive interventions for risk behavior reduction and 785 were assigned to control or comparison groups. Of all patients, $2264 \mathrm{did}$ not have any follow-up contact, 1949 had one follow-up contact, and 250 had 2 follow-up contacts, for a total of 2199 with follow-up; of those with follow-up, 1777 were intervention patients and 422 were in control or comparison groups. Comparisons of those with follow-up data with those with no follow-up data are shown in Table 2. Patients with at least one follow-up contact were older, less likely to be African American, more educated, had higher incomes, and were less likely to be smokers than patients with no follow-up contact (all $P<.05$ ).
The mean interval from baseline to final follow-up assessment varied across PBRNs, ranging from 31 days to 359 days (Table 3). Sample sizes for baseline and follow-up for each health behavior and network appear in Table 3.

\section{Outcomes}

Diet

After adjusting for age, sex, race/ethnicity, and education, diet scores improved significantly (decreased) from baseline to follow-up among intervention patients in 5 of the 7 networks (PBRNs 2, 4, 5, 8, and 9; $P<.05$; Figure 1). In sensitivity analyses using imputed follow-up data for study dropouts (assuming no improvement among dropouts), effects remained significant for all 5 networks that initially demonstrated improvement.

Table 2. Comparison of Patient Characteristics at Baseline for Those With and Without Follow-up Observations

\begin{tabular}{|c|c|c|c|}
\hline Baseline Patient Characteristics & $\begin{array}{l}\text { Follow-Up } \\
(\mathrm{n}=2199)\end{array}$ & $\begin{array}{l}\text { No Follow-Up } \\
\quad(\mathrm{n}=2264)\end{array}$ & $P$ \\
\hline \multicolumn{4}{|l|}{ Outcomes } \\
\hline Diet score & $5.66(2.57)$ & $5.58(2.54)$ & .2854 \\
\hline Physical activity (MET-minutes) & $2099(2735)$ & $2131(2672)$ & .7075 \\
\hline Average drinks per day & 0.3003 & 0.3336 & .3175 \\
\hline Current smokers & 26.2 & 32.0 & .0001 \\
\hline Unhealthy days & 25.4 & 22.0 & .0810 \\
\hline 0 & 38.7 & 38.5 & \\
\hline $1-13$ & 36.0 & 40.0 & \\
\hline \multicolumn{4}{|l|}{$\geq 14$} \\
\hline Women & 74.3 & 74.1 & .8866 \\
\hline Age (years) & $50.8(15.8)$ & $44.2(15.7)$ & $<.0001$ \\
\hline Race/ethnicity & & & $<.0001$ \\
\hline Non-Hispanic white & 62.4 & 56.7 & \\
\hline Non-Hispanic black & 11.6 & 19.2 & \\
\hline Hispanic/Latino & 20.9 & 18.9 & \\
\hline Other race & 5.1 & 5.2 & \\
\hline Education & & & $<.0001$ \\
\hline$<\mathrm{HS}$ & 14.0 & 12.3 & \\
\hline HS graduate/GED & 28.9 & 20.4 & \\
\hline$>\mathrm{HS}$ & 54.0 & 35.6 & \\
\hline Nonrespondent & 3.1 & 31.6 & \\
\hline Income (\$) & & & $<.0001$ \\
\hline$<25,000$ & 41.3 & 32.9 & \\
\hline $25,000-49,999$ & 21.6 & 12.3 & \\
\hline$\geq 50,000$ & 22.2 & 13.5 & \\
\hline Nonrespondent & 14.9 & 41.3 & \\
\hline BMI & $31.6(8.4)$ & $31.1(8.2)$ & .0527 \\
\hline
\end{tabular}

Values provided as mean (standard deviation) or \%.

Data from Prescription for Health: COMBO study.

BMI, body mass index; HS, high school; MET, metabolic equivalent. 
Table 3. Analytical Sample Sizes for Each Outcome by Practice-Based Research Network (PBRN) (Baseline/Follow-Up) and Health Risk Assessment Follow-Up Intervals

\begin{tabular}{ccccccc}
\hline PBRN ID & Diet & Physical Activity & Smoking & Alcohol & Quality of Life & Days to Final Follow-Up Assessment* \\
\hline 1 & $848 / 174$ & $871 / 172$ & $873 / 173$ & $850 / 105$ & $233 / 164$ & $186(43), 181$ \\
2 & $422 / 294$ & $421 / 294$ & $424 / 294$ & $421 / 292$ & $420 / 293$ & $177(47), 188$ \\
3 & $157 / 79$ & $155 / 76$ & $155 / 78$ & $0 / 0$ & $149 / 79$ & $292(70), 282$ \\
4 & $88 / 39$ & $87 / 39$ & $88 / 39$ & $88 / 39$ & $88 / 39$ & $158(44), 181$ \\
5 & $1573 / 872$ & $1514 / 849$ & $1568 / 849$ & $1512 / 869$ & $1237 / 695$ & $31(0), 31$ \\
8 & $386 / 236$ & $363 / 237$ & $405 / 236$ & $112 / 27$ & $344 / 231$ & $349(84), 359$ \\
9 & $88 / 45$ & $88 / 46$ & + & $87 / 45$ & $86 / 13$ & $199(62), 196$ \\
\hline
\end{tabular}

*Values shown as mean (standard deviation), median.

${ }^{\dagger}$ Smoking calculations for PBRN 9 were attempted but there were too few patients at follow-up.

\section{Physical Activity}

After adjusting for age, sex, race/ethnicity, improvement in physical activity was observed in 2 of the networks (PBRNs 4 and 5; $P<.05$; Figure 2). One network observed a decrease in physical activity over the average 349-day follow-up interval. In sensitivity analyses with imputed follow-up data for patients who dropped out of the study (assuming no improvement), none of the PBRNs demonstrated statistically significant improvement (all $P>.05$ ).

\section{Smoking}

Analysis of smoking outcomes was not possible in 2 networks because of the small sample size and too few smokers. Of the remaining 5 networks, the likelihood of being a current smoker was reduced significantly at follow-up for patients in the intervention in 2 PBRNs $(P<.05$; Figure 3$)$. These effects remained statistically significant in sensitivity analyses with imputed follow-up data for drop- outs, assuming dropouts continued smoking. Analysis of number of cigarettes per day yielded slightly different results, with statistically significant reduction for 2 PBRNs (PBRN 2, $P<.0001$; PBRN 5, $P=.0288)$.

\section{Alcobol}

Among the 6 PBRNs that collected data about alcohol use, 2 showed significant decrease in the average number of drinks consumed per day $(P<$ .05 ; Figure 4). These effects remained significant in sensitivity analysis with imputed follow-up data for dropouts, assuming no decrease among dropouts.

\section{Quality of Life}

There were significantly fewer unhealthy days at follow-up in 2 of the 7 networks in adjusted analyses (all $P<.05$; Figure 5); however, all interventions produced changes in the expected direction on quality of life measures. In sensitivity analysis,

Figure 1. Change in diet scores among intervention patients. PBRN, practice-based research network.

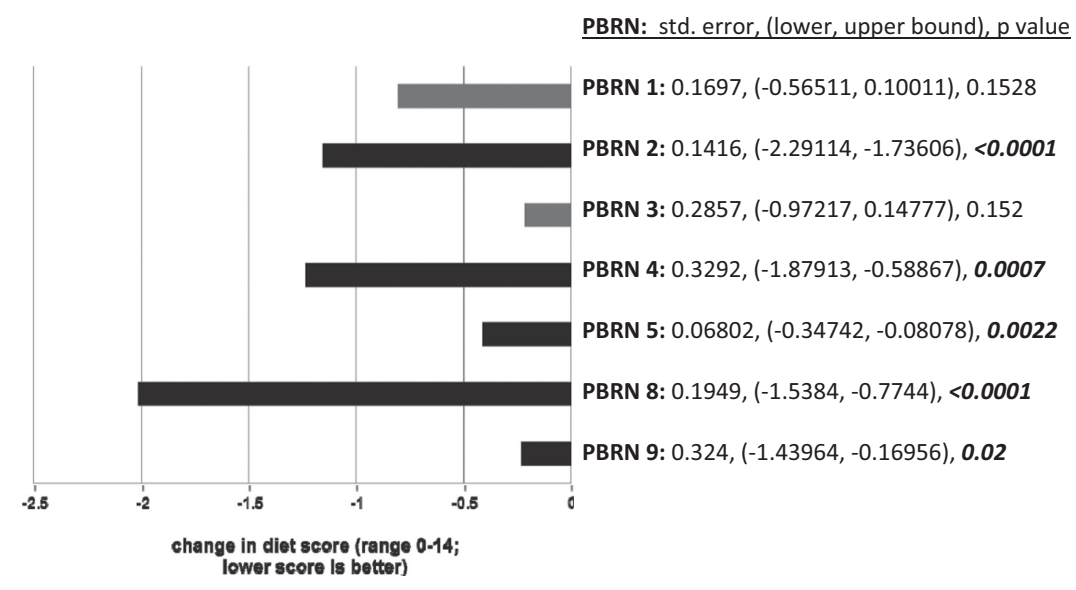


Figure 2. Change in physical activity among intervention patients. MET, metabolic equivalent; PBRN, practice-based research network.

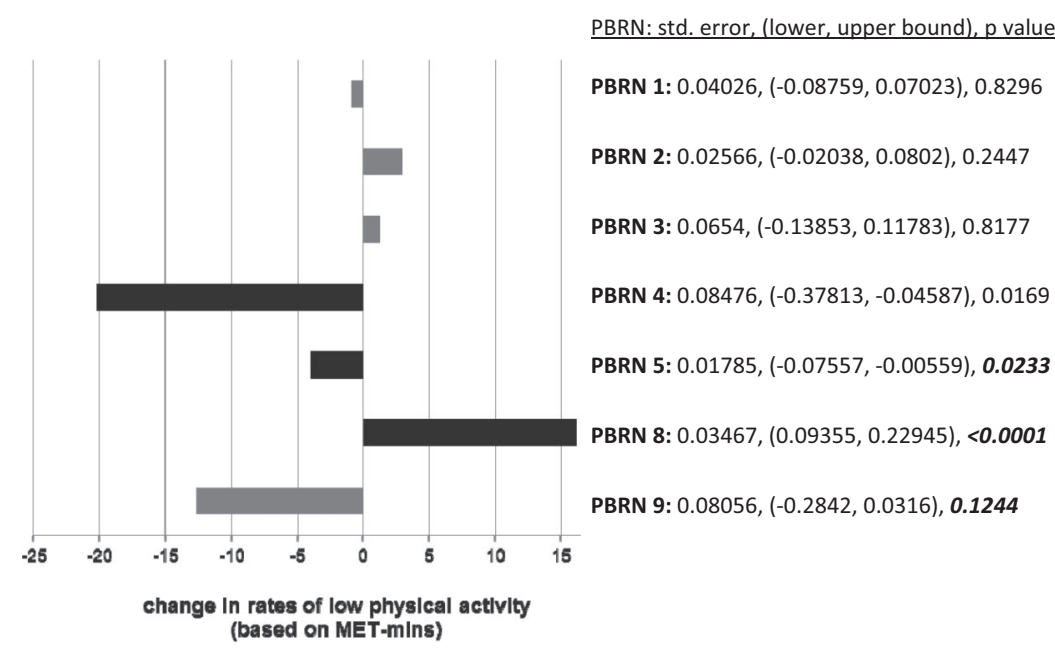

replacing missing data with imputed values for dropouts and assuming no improvement among dropouts, these results remained statistically significant for 2 PBRNs (PBRNs 2 and 8).

\section{Outcomes for PBRNs with Comparison Groups}

There were only limited data to assess the effects of the interventions by comparing intervention groups with comparison groups. Four PBRNs included data from comparison groups of patients. Direct comparison of change in intervention patients versus change in controls was statistically significant for only one PBRN (PBRN 8; $P=.0214$ ) on one health outcome (smoking), indicating greater reduction in smoking rates in the intervention group compared with con- trols. Although statistically significant changes among some control group patients were observed in diet scores (PBRNs 4, 8, and 9), physical activity scores (PBRN 4), and unhealthy days (PBRN 8), these did not differ significantly $(P>.05)$ from patients in the intervention groups.

\section{Discussion}

Although the overall results are somewhat encouraging, the COMBO study offered several important lessons for collaborative PBRN studies, particularly studies that address multiple health risk behaviors. With data from 54 practices in 7 PBRNs testing diverse interventions with practices in 11 states, the

Figure 3. Change in proportion of current smokers in intervention patients. PBRN, practice-based research network.

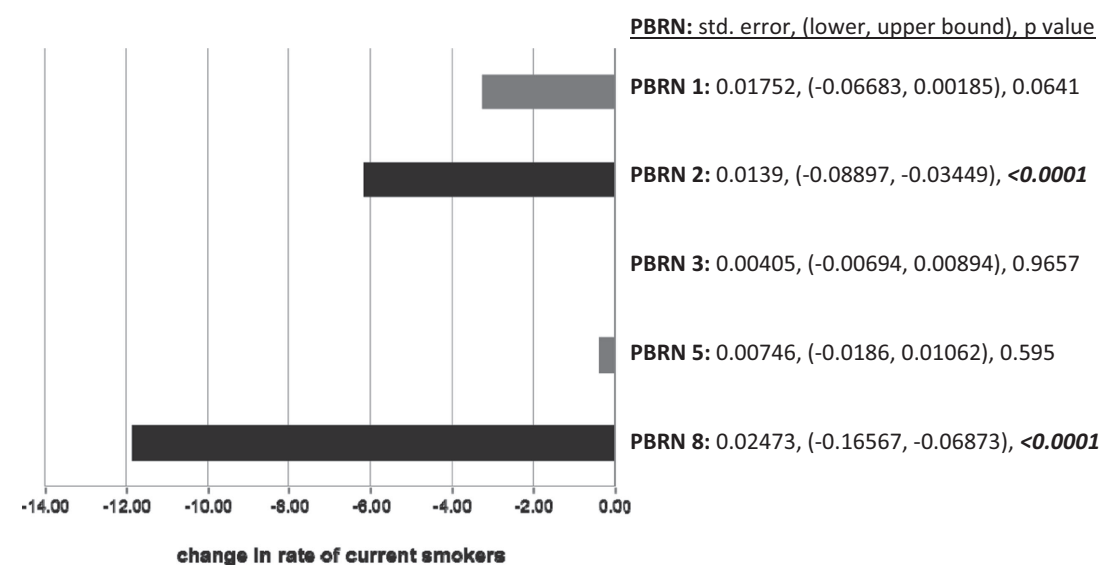


Figure 4. Change in average drinks per day in intervention patients. PBRN, practice-based research network.

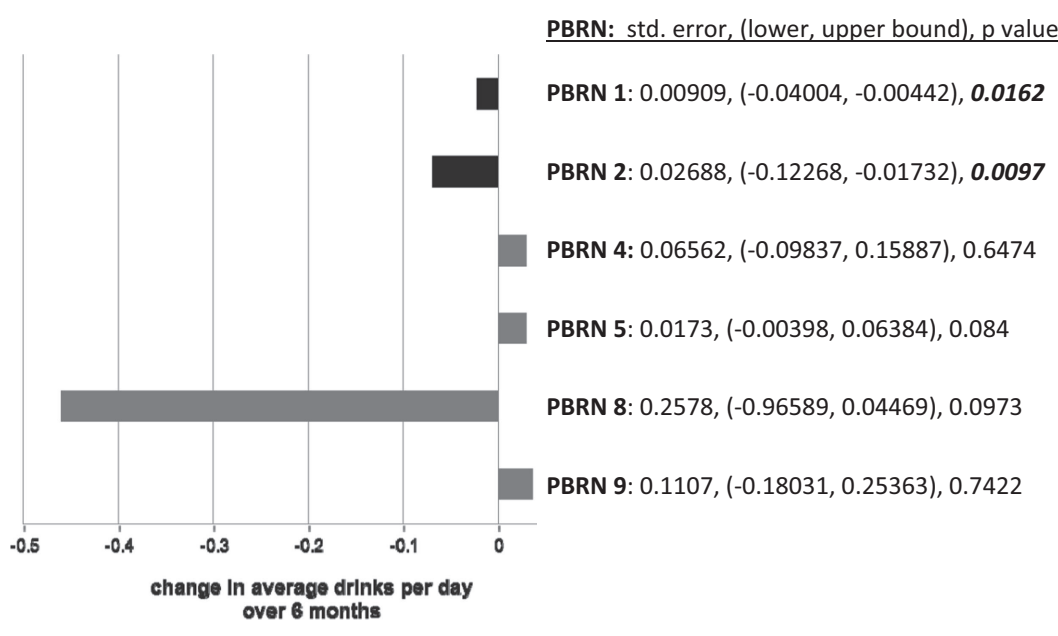

analysis showed that unhealthy behaviors generally improved for patients who participated in the interventions. A majority of networks showed statistically significant improvements in diet scores among intervention patients, whereas only 2 networks showed statistically significant improvements in physical activity, alcohol consumption, and rates of smokers. Although all networks reported a reduction in the number of unhealthy days (HRQOL) this was statistically significant in 2 of the networks. Since this COMBO PBRN study, other studies of multiple health risk behaviors also have found uneven and modest improvements across behaviors. ${ }^{8,12,14}$

\section{Methodologic Challenges}

The ambitious aims to standardize patient measures and to embed RE-AIM evaluation principles

across 7 PBRNs were intended to yield a better understanding of the results. In fact, this amplified our understanding of limitations in the instrumentation, project implementation, and interpretation of the outcomes.

\section{Instrumentation}

Despite careful attention to selection of common data collection instruments, the utility of these measures in routine primary care proved challenging. The baseline data identified potential problems with data on some of the instruments (especially the International Physical Activity Questionnaire), including missing data, instrument complexity, and general difficulties addressing problem drinking in primary care. ${ }^{17}$ Although some patients may have completed some of the health behavior questions,

Figure 5. Change in number of unhealthy days in intervention patients. PBRN, practice-based research network.

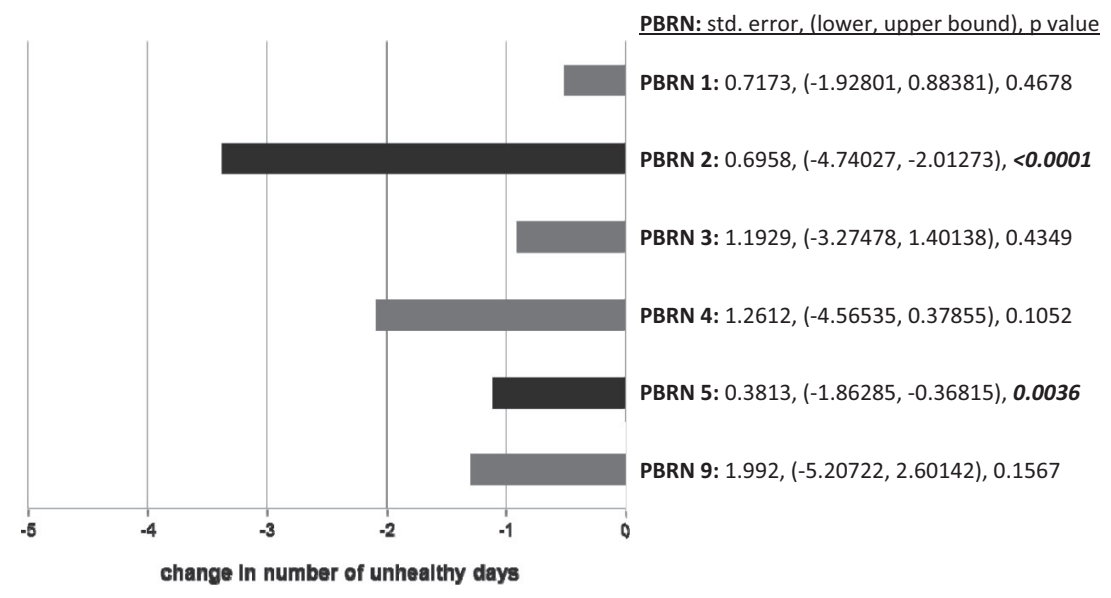


without complete instruments (items missing), outcome variables could not be computed. The problems of missing item-level data meant that even fewer cases were likely to be included in the analysis at follow-up, which was further compounded by low follow-up rates in some networks. Simpler instruments, such as the diet questionnaire, may help to avoid these problems in future work.

\section{Implementation}

There was optimism early in the design of the COMBO study that some level of cross-PBRN comparative analysis would be possible based on our understanding of similarities in the study designs of the individual PBRNs. As our understanding of the projects grew during implementation and data reporting, the prospects for such comparative analysis grew dim. The program office and evaluation team required projects to help document implementation of their interventions. Their reports helped illuminate how difficult real-world, practical trials can be. ${ }^{21,29}$ Other Prescription for Health investigators noted that implementation of interventions were uneven and difficult in some networks. ${ }^{30,31}$ Although the ability to implement an intervention is important to consider when evaluating the utility of an intervention in practices, it was not possible in this study to assess how implementation variance affected the outcomes or how to interpret positive findings.

\section{Interpretation}

Among the 4 health behaviors, dietary scores stood out in terms of both significant improvements and better measurement. Furthermore, findings regarding dietary improvement were robust even when assuming no improvement among patients with no follow-up data. Yet there are further lessons concerning the interpretation of the results. The relative success of changes in diet versus the other health behaviors may have been because of the differential effects of the intervention, the difficulty of change over the follow-up period for other health behaviors, the relative sensitivity to change of the different instruments, or the relative ease of imputing missing item-level data for diet scores compared with other health behavior outcome data. The present study design did not allow us to distinguish among these explanations. Although the results here are consistent with other evidence using the same instrument, ${ }^{32}$ the clinical significance of the findings are unclear. Individual diets improved overall (Figure 1). When statistically significant, patients improved between .5 and 2 points. This could mean, for example, that they reduced their frequency of eating snacks, chips, or fast food from 4 or more times per week to 3 or fewer times, or from 2 to 3 times per week to 1 or fewer. It could mean they are increasing the frequency of servings of vegetables or healthy proteins. The instrument itself is designed for "starting the conversation" with patients and hence may be more effective on an individual counseling basis than for understanding specific practice- or project-level effects.

The length of follow-up also played a role in the data we analyzed. Figure 3 displays the change in rates of current smokers. This means that patients had not smoked a cigarette in the past 30 days. In one network with significant improvement (PBRN 2) the follow-up was at about 6 months; in the other (PBRN 8) the follow-up was at about 11 months. We do not know if the nonsmoking status was sustained beyond this or what exactly happened between baseline and follow-up. Furthermore, in PBRN 8's analysis, improvement in smoking rates among intervention patients was not large enough to demonstrate statistically significant difference from their comparison group.

Even less clear are the outcomes of problem drinking (Figure 4). The 2 networks showing statistically significant decreases in the average drinks consumed per day was less than one tenth of a drink. On an individual patient level, it is not known if this contributes meaningfully to a sustained reduction in drinking to a range outside of heavy or problem drinking.

\section{Variation in Practice Population and Performance}

Overall, the proportion of cases with follow-up data were less than $50 \%$ and those with follow-up data were older, were less likely to be African American, were more educated, had higher incomes, and were less likely to be smokers. It is clear that although many patients initially enrolled in the interventions, many did not have continued engagement with the PBRN study. The analysis may represent types of patients who are more likely to maintain engagement with health behavior interventions, more likely to respond to follow-up surveys, or simply more likely to return for follow-up visits in routine primary care, and this sample of patients may not be generalizable to all primary 
care practices in the United States. These data were self-reported and are subject to overreporting or underreporting, especially the potential overreporting of physical activity. ${ }^{33}$ Differences in data collection may have led to unknown differences in the data across networks. Several networks have reported results based on their own analyses of their network's data. ${ }^{30,34,35}$ Because our analysis used different statistical methods and different outcome variable definitions, findings of specific health behavior outcomes may be different.

\section{Recommendations}

Even after choosing common measures, cross-network studies that aim to combine data from across PBRN-tailored interventions could benefit from (1) a requirement of standardized data collection methods, (2) standardized follow-up periods, (3) an emphasis on retaining patients in the study even if they choose to not participate in the intervention, and (4) inclusion of control groups.

\section{Conclusions}

Although some patients in these 7 PBRNs improved in several health behaviors and quality of life, the strength of evidence for field-ready methods to address multiple health risk behaviors remains elusive. The use of common measures to assess changes in 4 unhealthy behaviors was practically achieved in PBRNs testing diverse strategies to improve behaviors. Nonetheless, variations in implementation, instrumentation performance, and some features of study designs overwhelmed potential cross-PBRN comparisons. Primary care interventions and instrumentation that address diet seem to be the most promising for demonstrating change over time among patients. For common measures to be useful to compare across practices or PBRNs, greater standardization of study designs and careful attention to practicable implementation strategies are necessary.

\section{References}

1. Berrigan D, Dodd K, Troiano RP, Krebs-Smith SM, Barbash RB. Patterns of health behavior in US adults. Prev Med 2003;36(5):615-23.

2. Coups EJ, Gaba A, Orleans CT. Physician screening for multiple behavioral health risk factors. Am J Prev Med 2004;27(2 Suppl 1):34-41.

3. Goldstein MG, Whitlock EP, DePue J. Multiple behavioral risk factor interventions in primary care: Summary of research evidence. Am J Prev Med 2004;27(2 Suppl 1):61-79.
4. Pronk NP, Anderson LH, Crain AL, et al. Meeting recommendations for multiple healthy lifestyle factors: prevalence, clustering, and predictors among adolescent, adult, and senior health plan members. Am J Prev Med 2004;27(2 Suppl 1):25-33.

5. Pronk NP, Peek CJ, Goldstein MG. Addressing multiple behavioral risk factors in primary care. A synthesis of current knowledge and stakeholder dialogue sessions. Am J Prev Med 2004;27(2 Suppl):4-17.

6. Fine LJ, Philogene GS, Gramling R, Coups EJ, Sinha S. Prevalence of multiple chronic disease risk factors: 2001 National Health Interview Survey. Am J Prev Med 2004;27(2 Suppl 1):18-24.

7. Prochaska JO, Butterworth S, Redding CA, et al. Initial efficacy of MI. TTM tailoring and HRI's with multiple behaviors for employee health promotion. Prev Med 2008;46(3):226-31.

8. Vandelanotte C, Reeves MM, Brug J, Bourdeaudhuij ID. A randomized trial of sequential and simultaneous multiple behavior change interventions for physical activity and fat intake. Prev Med 2008;46(3): 232-7.

9. Werch CE (Chad), Moore MJ, Bian H, et al. Are effects from a brief multiple behavior intervention for college students sustained over time? Prev Med 2010;50(1-2):30-4.

10. Koelewijn-van Loon MS, Der Weijden $T$ van, Ronda G, et al. Improving lifestyle and risk perception through patient involvement in nurse-led cardiovascular risk management: a cluster-randomized controlled trial in primary care. Prev Med 2010; 50(1-2):35-44.

11. Johnson SS, Paiva AL, Cummins CO, et al. Transtheoretical Model-based multiple behavior intervention for weight management: effectiveness on a population basis. Prev Med 2008;46(3):238-46.

12. Krist AH, Woolf SH, Johnson RE, et al. Patient costs as a barrier to intensive health behavior counseling. Am J Prev Med 2010;38(3):344-8.

13. Eakin E, Reeves M, Lawler S, et al. Telephone counseling for physical activity and diet in primary care patients. Am J Prev Med 2009;36(2):142-9.

14. Spink KS, Wilson KS. Physician counseling and longer term physical activity. J Prim Care Community Health. 2010;1(3):173-7.

15. Morabia A, Costanza MC. Multiple health behavior change interventions: tell us what you see. Prev Med 2010;50(1-2):1-2.

16. Prochaska JO. Multiple health behavior research represents the future of preventive medicine. Prev Med 2008;46(3):281-5.

17. Fernald DH, Froshaug DB, Dickinson LM, et al. Common measures, better outcomes (COMBO): a field test of brief health behavior measures in primary care. Am J Prev Med 2008;35(5 Suppl): S414-22.

18. Cifuentes M, Fernald DH, Green LA, et al. Prescription for health: changing primary care practice 
to foster healthy behaviors. Ann Fam Med 2005; 3(Suppl 2):S4-11.

19. Wagner EH, Austin BT, Von Korff M. Organizing care for patients with chronic illness. Milbank Q 1996;74(4):511-44.

20. Glasgow RE, Vogt TM, Boles SM. Evaluating the public health impact of health promotion interventions: the RE-AIM framework. Am J Public Health 1999;89(9):1322-7.

21. Etz RS, Cohen DJ, Woolf SH, et al. Bridging primary care practices and communities to promote healthy behaviors. Am J Prev Med 2008;35(5 Suppl): S390-7.

22. Dodoo MS, Krist AH, Cifuentes M, Green LA Start-up and incremental practice expenses for behavior change interventions in primary care. Am J Prev Med 2008;35(5 Suppl):S423-30.

23. Centers for Disease Control and Prevention, $\mathrm{Na}-$ tional Center For Chronic Disease Prevention and Health Promotion. Behavioral Risk Factor Surveillance System [homepage]. Updated 24 February 2012. Available from: http://www.cdc.gov/brfss/. Accessed March 13, 2012.

24. Glasgow RE, Ory MG, Klesges LM, Cifuentes M, Fernald DH, Green LA. Practical and relevant selfreport measures of patient health behaviors for primary care research. Ann Fam Med 2005;3(1):73-81.

25. Gaskins ND, Sloane PD, Mitchell CM, Ammerman A, Ickes SB, Williams CS. Poor nutritional habits: a modifiable predecessor of chronic illness? A North Carolina Family Medicine Research Network (NC-FM-RN) study. J Am Board Fam Med 2007;20(2):124-34.

26. Craig CL, Marshall AL, Sjöström M, et al. International physical activity questionnaire: 12 -country reliability and validity. Med Sci Sports Exerc 2003; 35(8):1381-95.
27. Moriarty DG, Kobau R, Zack MM, Zahran HS. Tracking healthy days-a window on the health of older adults. Prev Chronic Dis 2005;2(3):A16.

28. Bryk S, Raudenbush A. Hierarchical linear models: applications and data analysis methods. 2nd ed. Thousand Oaks, CA: Sage Publications; 2002.

29. Cohen DJ, Crabtree BF, Etz RS, et al. Fidelity versus flexibility: translating evidence-based research into practice. Am J Prev Med 2008;35(5 Suppl): S381-9.

30. Ferrer RL, Mody-Bailey P, Jaén CR, Gott S, Araujo S. A medical assistant-based program to promote healthy behaviors in primary care. Ann Fam Med 2009;7(6):504-12.

31. Cohen DJ, Balasubramanian BA, Isaacson NF, et al. Coordination of health behavior counseling in primary care. Ann Fam Med 2011;9(5):406-15.

32. Paxton AE, Strycker LA, Toobert DJ, Ammerman AS, Glasgow RE. Starting the conversation performance of a brief dietary assessment and intervention tool for health professionals. Am J Prev Med 2011; 40(1):67-71.

33. Rzewnicki R, Vanden Auweele Y, De Bourdeaudhuij I. Addressing overreporting on the International Physical Activity Questionnaire (IPAQ) telephone survey with a population sample. Pub Health Nutr 2003;6(3):299-305.

34. Holtrop JS, Dosh SA, Torres T, Thum YM. The community health educator referral liaison (CHERL): a primary care practice role for promoting healthy behaviors. Am J Prev Med 2008;35(5 Suppl):S365-72.

35. Olson AL, Gaffney CA, Lee PW, Starr P. Changing adolescent health behaviors: the healthy teens counseling approach. Am J Prev Med 2008;35(5 Suppl): S359-64. 